\title{
Lévy Process, Proportional Transaction Costs and Foreign Exchange
}

\author{
Obonye Doctor $^{1}$, Elias R. Offen ${ }^{1,2} \&$ Edward M. Lungu ${ }^{2}$ \\ ${ }^{1}$ School of Sciences, Botswana International University of Science and Technology, Palapye, Botswana \\ ${ }^{2}$ School of Sciences, Botswana International University of Science and Technology, Palapye, Botswana \\ Correspondence: Elias R. Offen, Faculty of Science, University of Botswana, Gaborone, Botswana. \\ E-mail: elias.offen@gmail.com
}

Received: June 21, 2017 Accepted: July 8, 2017 Online Published: September 27, 2017

doi:10.5539/jmr.v9n5p133 URL: https://doi.org/10.5539/jmr.v9n5p133

\begin{abstract}
We analyse optimal portfolio selection problem of maximizing the utility of an agent who invests in a stock and money market account in the presence of proportional transaction cost $\lambda>0$ and foreign exchange rate. The stock price follows a (generalized) Geometric Itô-Lévy process. The utility function is $U(c)=c^{p} / p$ for all $c \geq 0, p<1, p \neq 0$.
\end{abstract}

Keywords: Itô-Lévy diffusions, Portfolio optimization, foreign exchange, stochastic control, utility functions

\section{Introduction}

The study of dynamic portfolio choice has a long history, tracking back to Merton (1971). The introduction of the jumps to the Merton model where the dynamics of stock price are modelled by a Lévy process has attracted a lot of researchers; $\varnothing \mathrm{ksendal}$ and Sulem $(2004,2005,2014)$, Tankov (2005) and many more. But so far when the jumps are included, the determination of the optimal portfolio has not been amenable to a closed-form solution and this is a long-standing open problem in continuous time finance. Lévy processes were introduced by Lévy (1937) who pioneered the theory of infinite divisibility. Modern accounts of the probability theory of Lévy processes are given in Sato (1999) and Applebaum (2004).

Our work is motivated by Øksendal and Sulem (2014) on their quick introduction to some important tools in the modern research within mathematical finance, with emphasis on applications to portfolio optimization and risk minimization, Liu (2004) by successfully analyzing and deriving the optimal transaction policy in an explicit form when $n \geq 1$ risky assets are correlated and subject to fixed transaction costs in an infinite horizon, Janeček and Shreve (2004) and Bichuch (2011) by considering the cases where an agent invests in a stock and a money market account with the hope of maximizing his/her wealth in an infinite time horizon and at the final time $T$, respectively, in the presence of the proportional transaction costs. Their main idea were to provide a heuristic and a rigorous derivation of the value function in powers of $\lambda^{\frac{1}{3}}$. In this paper, we intend to make contribution in modeling choices and bring powerful yet simple mathematical tools through discussing the impact of foreign exchange when the risky asset is a generalised Itô-Lévy process in the face of transaction costs. We discuss several propositions based on different kinds of jumps in our optimal portfolio problem.

The rest of the paper is organised as follows. In section 2, we present in detail out model. In section 3, we discuss the optimal portfolio in the absence of the transaction costs. In section 4 , we provide an optimal investment in the presence of transaction costs. Conclusions are in section 5 followed by references.

\section{The Model}

We consider a market consisting of two investment opportunities, the money market account a bond and a stock. Assume that there are two riskless assets BW money market and US money market in BW pula and US dollars, respectively with interest rates $r_{1}>0$ and $r_{2}>0$. Due to uncertainty about the future exchange rates, the asset in a US money market is not riskless with respect to the BW pula investor, nor is the asset in BW pula market riskless with respect to the US dollar investor, thus choice of numeraire determines which asset is riskless. Let $D_{t}$ be the rate of exchange at time $t \in[0, T]$. The dynamics of $D_{t}$ at time $t \in[0, T]$ follow the diffusion process.

$$
d D_{t}=D_{t}\left[\mu_{1} d t+\sigma_{1} d B_{1}(t)\right]
$$

where $B_{1}(t)$ is the Brownian motion. Let bond share price $R_{t}^{b}$ at time $t \in[0, T]$ reported in units of bond be given by

$$
d R_{t}^{b}=r_{1} R_{t}^{b} d t, R_{0}^{b}=1,
$$

Thus, the share price of the money market at time $t$ in dollar is $R_{t}^{b} D_{t}=R_{t}$. From equation (1) and (2) we obtain the stochastic differential equation (SDE) given by

$$
d R_{t}=R_{t}\left[\left(r_{1}+\mu_{1}\right) d t+\sigma_{1} d B_{1}(t)\right], \quad R(0)=r_{0}>0 .
$$


In the point of view of the bond investor; in the bond markets, neither the bond nor the exchange rate is tradable. However, $\left\{R_{t}\right\}_{t \geq 0}$ can be thought as a bond tradable. Suppose the share price of stock, $S_{t}$, which is in dollars at any time $t \geq 0$ is a geometric Itô-Lévy process given by

$$
d S_{t}=S_{t-}\left[\mu_{2} d t+\sigma_{2} d B_{2}(t)+\int_{\mathbb{R}} g(t, \zeta) \tilde{N}(d t, d \zeta)\right] ; \quad S(0)=S_{0}>0 .
$$

Taking $g>-1$ then $S(t)$ can never jump to 0 or a negative value (Øksendal and Sulem, 2014). Solution to (3) is given by

$$
S_{t}=S_{0} \exp \left\{\left(\mu_{2}-\frac{\sigma_{2}^{2}}{2}\right) t+\sigma_{2} B_{2}(t)+\int_{0}^{t} \int_{\mathbb{R}}(\ln (1+g)-g) v(d \zeta) d s+\int_{0}^{t} \int_{\mathbb{R}} \ln (1+g) \tilde{N}(d t, d \zeta)\right\}
$$

where $\mu_{1,2}>r_{1,2}>0$ and $\sigma_{1,2}>0$ are (constants) known as mean rates of return and volatilities of the exchange rate and stock, respectively. $\tilde{N}$ denote the compensated jump measure of $S$, defined as $\tilde{N}(d t, d \zeta)=N(d t, d \zeta)-v(d \zeta) d t, N(d t, d \zeta)$ is the differential notation of the random measure $N([0, t], A)$. $\zeta$ is regarded as a generic jump size. The jump measure $N([0, t], A)$ gives the number of jumps of $S$ up to time $t$ with jump size in the set $A \subset \mathbb{R}-\{0\}$. Moreover, if we assume that $\bar{A} \subset \mathbb{R}-\{0\}$, then it can be shown that $A$ contains only finitely many jumps in any finite time interval $(\varnothing \mathrm{ksendal}$ and Sulem, 2014). The Lévy measure $v(\cdot)$ of $S$ is defined by $v(A)=\mathbb{E}[N([0,1], A)]$ and satisfies $\int_{\mathbb{R}} \min \{1,|x|\} \nu(d x)<\infty, \operatorname{so}$ the jumps have finite variation. $S_{t-}$ means that whenever there is a jump, the value of the process before the jump is used on the left-hand side of the formula. $\left\{B_{i}(t)\right\}_{t \geq 0}$ for $i=1,2$ is the standard Brownian motion on a filtered probability space $\left(\Omega, \mathcal{F},\left(\mathcal{F}_{t}\right)_{t \geq 0}, \mathbb{P}\right)$ with $B_{i}(0)=0$ for $i=1,2$ almost surely. The correlation between $\left\{B_{1}(t)\right\}_{t \geq 0}$ and $\left\{B_{2}(t)\right\}_{t \geq 0}$ is given by

$$
B_{1}(t)=\beta B_{2}(t)+\sqrt{1-\beta^{2}} B_{t}, \quad-1 \leq \beta \leq 1,
$$

where $\beta$ is the correlation coefficient and $B_{t}$ is a Brownian motion independent of $B_{2}(t)$.

Define the dynamics of the bond and stock holdings in monetary terms as

$$
\begin{gathered}
d X_{t}=\left(r_{1}+\mu_{1}\right) X_{t} d t+\sigma_{1} X_{t} d B_{1}(t)-(1+\lambda) d L_{t}+(1-\lambda) d M_{t}, \quad X_{0}=x, \\
d Y_{t}=Y_{t-}\left[\mu_{2} d t+\sigma_{2} d B_{2}(t)+\int_{\mathbb{R}} g(t, \zeta, \omega) \tilde{N}(d t, d \zeta)\right]+d L_{t}-d M_{t}, \quad Y_{0}=y,
\end{gathered}
$$

where $\lambda \in[0,1)$ accounts for proportional transaction costs paid from the money market, $L_{t}$ represents the cumulative dollar value of stock purchased up to time $t$, while $M_{t}$ is the cumulative dollar value of stock sold. The agent must choose a policy consisting of two adapted processes $L_{t}$ and $M_{t}$ that are nondecreasing and right-continuous with left limits and $L_{0-}=M_{0-}=0$. Note that, purchase of $d L_{t}$ units of stock requires a payment of $(1+\lambda) d L_{t}$ from the money market account while sale of $d M_{t}$ units of stock realizes only $(1-\lambda) d M_{t}$ in cash. The investor's net wealth in monetary terms at time $t \in[0, T]$ is

$$
W_{t}=X_{t}+Y_{t}-\lambda\left|Y_{t}\right|
$$

Define the solvency region

$$
Q_{\lambda}:=\{(x, y) ; x+(1+\lambda) y \geq 0, x+(1-\lambda) y \geq 0\}
$$

as the set of positions from which the agent can move to a positive wealth in both assets. The policy $\left(L_{s}, M_{s}\right)_{s \in[t, T]}$ is admissible for the initial position $(t, x, y)$ if $\left(X_{s}, Y_{s}\right)$ starting from $\left(X_{t-}, Y_{t-}\right)=(x, y)$ and given (5) and (6) in $\overline{Q_{\lambda}} \forall s \in[t, T]$. Since the agent may choose to re-balance his position, then we set the initial time to be $t-$. We let $\mathcal{A}(t, x, y)$ be the set of all such admissible policies.

Define the agent's utility function by $U(c)=\frac{c^{p}}{p}$ for all $c \geq 0, p<1, p \neq 0$. Define the value function as the supremum of the utility of the final cash position, after the agent liquidates her stock holdings

$$
v(t, x, y)=\sup _{(L, M) \in \mathcal{A}(t, x, y)} \mathbb{E}^{x, y}\left[U\left(X_{T}+Y_{T}-\lambda\left|Y_{T}\right|\right) \mid \mathcal{F}_{t}\right]
$$

for $(t, x, y) \in[0, T] \times \overline{Q_{\lambda}}$. Define the auxiliary value function

$$
v_{\rho}(t, x, y)=\sup _{(L, M) \in \mathcal{A}(t, x, y)} \mathbb{E}^{x, y}\left[e^{-\rho(T-t)} U\left(X_{T}+Y_{T}-\lambda\left|Y_{T}\right|\right) \mid \mathcal{F}_{t}\right]
$$

for $(t, x, y) \in[0, T] \times \overline{Q_{\lambda}}$ and $\rho \geq 0$. Here $\rho \geq 0$ is the discounting factor and $\mathbb{E}^{x, y}$ denotes the conditional expectation at time $t$ given that initial endowment is $X_{t}=x$ and $Y_{t}=y$. Therefore

$$
v(t, x, y)=e^{\rho(T-t)} v_{\rho}(t, x, y), \rho \geq 0,(t, x, y) \in[0, T] \times \overline{Q_{\lambda}} .
$$


The paper concentrates on finding $v_{\rho}$. The problem with foreign exchange rate, jumps and no transaction costs $(\lambda=0)$ has solution

$$
v_{\rho}(t, x, y)=\frac{1}{p} e^{p A(T-t)}(x+y)^{p}, \rho \geq 0,(t, x, y) \in[0, T] \times \overline{\mathcal{S}_{\lambda}},
$$

where

$$
\begin{aligned}
A & =-\frac{\rho}{p}+\mu+\frac{(p-1)}{2}\left(\sigma_{1}^{2}-\pi^{\star 2}\left(\sigma_{1}^{2}+\sigma_{2}^{2}\right)\right) \\
& +\frac{1}{p} \int_{\mathbb{R}}\left\{\left(1+\pi^{\star} g(t, \zeta)\right)^{p}-1+p \pi^{\star} g(t, \zeta)\left((p-1) \pi^{\star} g(t, \zeta)-1\right)\right\} v(d \zeta)
\end{aligned}
$$

and the wealth proportion satisfies the equation

$$
\pi^{\star}=\frac{\mu-\mu_{2}+(p-1) \sigma_{1}^{2}}{(p-1)\left(\sigma_{1}^{2}+\sigma_{2}^{2}\right)}+\left((p-1)\left(\sigma_{1}^{2}+\sigma_{2}^{2}\right)\right)^{-1} \int_{\mathbb{R}} g(t, \zeta)\left[\left(1+\pi^{\star} g(t, \zeta)\right)^{p-1}-1\right] v(d \zeta)
$$

with $\mu=r_{1}+\mu_{1}$.

\section{No Transaction Costs Case}

Let $\pi(t)$ denote a portfolio representing the portion of the total wealth invested in the risky asset at time $t \in[0, T]$. If we assume that $\pi(t)$ is self-financing, the corresponding wealth $W(t)=W_{\pi}(t)$ satisfies

$$
d W_{t}=W_{t}\left[\left(\mu_{2} \pi+(1-\pi) \mu\right) d t+(1-\pi) \sigma_{1} d B_{1}(t)+\pi \sigma_{2} d B_{2}(t)+\pi \int_{\mathbb{R}} g(t, \omega) \tilde{N}(d t, d \zeta)\right],
$$

where $\mu=r_{1}+\mu_{1}$. We seek to maximize $\mathbb{E}\left[e^{-\rho(T-t)} U\left(W_{\pi}(T)\right) \mid \mathcal{F}_{t}\right]$ over all $\pi \in \mathcal{A}$, where $\mathcal{A}$ denotes the set of all admissible portfolios and $U$ is the power utility function.

From Theorem 3.2 in Øksendal and Sulem (2014) and taking $U(w)=w^{p} / p, p<1, p \neq 0$. Then the problem is to $\operatorname{maximize} \mathbb{E}\left[e^{-\rho(T-t)}\left(W_{\pi}(T)\right)^{p} / p \mid \mathcal{F}_{t}\right]$. Thus,

$$
\begin{aligned}
(\mathcal{L} f)(t, w)=\frac{\partial f}{\partial t}(t, w) & +w\left(\left(\mu_{2} \pi+(1-\pi) \mu\right)\right) \frac{\partial f}{\partial w}(t, w)+\frac{1}{2} w^{2}\left[(1-\pi)^{2} \sigma_{1}^{2}+\pi^{2} \sigma_{2}^{2}\right] \frac{\partial^{2} f}{\partial w^{2}}(t, w) \\
& +\int_{\mathbb{R}}\{f(t, w+w \pi g(t, \zeta))-f(w)-\nabla f(w) w \pi g(t, \zeta)\} v(d \zeta)=0 .
\end{aligned}
$$

We guess that $f(t, w)=e^{-\rho t}(h(t) w)^{p} / p$, where $h(t)$ is a deterministic function. We also assume that $g(t, z)$ is deterministic. Then the optimal portfolio $\pi^{\star}$ is the solution of the equation

$$
\pi^{\star}=\frac{\mu-\mu_{2}+(p-1) \sigma_{1}^{2}}{(p-1)\left(\sigma_{1}^{2}+\sigma_{2}^{2}\right)}+\left((p-1)\left(\sigma_{1}^{2}+\sigma_{2}^{2}\right)\right)^{-1} \int_{\mathbb{R}} g(t, \zeta)\left[\left(1+\pi^{\star} g(t, \zeta)\right)^{p-1}-1\right] v(d \zeta) .
$$

Now, if we assume that the measure $v$ has light tails, then we can apply Taylor expansion

$$
1-\left(1+\pi^{\star} g(t, \zeta)\right)^{-(1-p)}=(1-p) \pi^{\star} g(t, \zeta)+o\left(g(t, \zeta)^{2}\right)
$$

which leads to the optimal portfolio (13) evolving as

$$
\pi^{\star} \approx \frac{1}{p-1} \cdot \frac{\mu-\mu_{2}+(p-1) \sigma_{1}^{2}}{\left(\sigma_{1}^{2}+\sigma_{2}^{2}\right)-\int_{\mathbb{R}} g^{2}(t, \zeta) v(d \zeta)} .
$$

We note that for pure diffusive case, $v=0$, we obtain the Merton solution

$$
\pi^{\star}=\frac{\mu-\mu_{2}+(p-1) \sigma_{1}^{2}}{(p-1)\left(\sigma_{1}^{2}+\sigma_{2}^{2}\right)} .
$$

Therefore, with jumps it is optimal to place a larger wealth fraction in the risky investment. The resulting value is smaller than in the no-jump case (see Øksendal and Sulem (2005)). Next, we find $h(t)$ (our assumed solution of (13)) by substituting corresponding derivatives into (13) and setting $h(T)=1$ to get

$$
h(t)=\exp \{A(T-t)\}
$$


where

$$
\begin{aligned}
p A=-\rho & +p\left(\mu_{2} \pi^{\star}+\left(1-\pi^{\star}\right) \mu\right)+\frac{p(p-1)}{2}\left(\left(1-\pi^{\star}\right)^{2} \sigma_{1}^{2}+\pi^{\star^{2}} \sigma_{2}^{2}\right) \\
& +\int_{\mathbb{R}}\left\{\left(1+\pi^{\star} g(t, \zeta)\right)^{p}-1-p \pi^{\star} g(t, \zeta)\right\} v(d \zeta) \\
& =-\rho+p \mu+\frac{p(p-1)}{2}\left(\sigma_{1}^{2}-\pi^{\star 2}\left(\sigma_{1}^{2}+\sigma_{2}^{2}\right)\right) \\
& +\int_{\mathbb{R}}\left\{\left(1+\pi^{\star} g(t, \zeta)\right)^{p}-1+p \pi^{\star} g(t, \zeta)\left((p-1) \pi^{\star} g(t, \zeta)-1\right)\right\} v(d \zeta) .
\end{aligned}
$$

Finally, the value function is given by

$$
f(t, w)=v(t, w)=e^{-\rho t} e^{p A(T-t)} w^{p} / p, \quad p<1, \quad p \neq 0 .
$$

Substituting the constants $\pi^{\star}$ and $A$ in (12), yields the optimal wealth process $W_{t}^{\star}$ of the Ito-Lévy process.

\section{Optimal Investment in the Presence of Transaction Costs}

Theorem 1 Since $X, Y$ are jointly Markov, the value function $v(t, x, y)$ defined by equation (8) is a viscosity solution of the Hamilton-Jacobi-Bellman $(H J B)$ equation on $[0, T] \times Q_{\lambda}$

$$
\min \left\{-v_{t}-\mathcal{K}(v),-(1-\lambda) v_{x}+v_{y},(1+\lambda) v_{x}-v_{y}\right\}=0
$$

where $\mathcal{K}$ is the second-order differential operator given by

$$
\begin{aligned}
\mathcal{K}(v) & =-\rho v(t, x, y)+\frac{1}{2}\left[\sigma_{2}^{2} y^{2} v_{y y}(t, x, y)+\sigma_{1}^{2} x^{2} v_{x x}(t, x, y)\right] \\
& +\mu x v_{x}(t, x, y)+\mu_{2} y v_{y}(t, x, y)+\beta \sigma_{1} \sigma_{2} x y v_{x y}(t, x, y) \\
& -\int_{\mathbb{R}}\left\{v(t, x, y+g(y, \zeta))-v(t, x, y)-v_{y}(t, x, y) g(y, \zeta)\right\} v(d \zeta)
\end{aligned}
$$

with the terminal condition

$$
v(T, x, y)=U(x+y-\lambda|y|), \quad(x, y) \in \overline{Q_{\lambda}} .
$$

Noting that the value function $v$ has the homotheticity property for $\gamma>0$,

$$
v(t, \gamma x, \gamma y)=\gamma^{p} v(t, x, y)
$$

where the admissible policy $\mathcal{A}(t, \gamma x, \gamma y)=\{(\gamma L, \gamma M):(L, M) \in \mathcal{A}(t, x, y)\}$. We can reduce the problem into that of two variables with the transformation; $\xi=\frac{y}{x+y}, 1-\xi=\frac{x}{x+y}$. Define

$$
\phi(t, \xi)=v(t, 1-\xi, \xi), \quad(t, \xi) \in[0, T] \times \overline{Q_{\phi}}
$$

where $\overline{Q_{\phi}}=\left[\frac{-1}{\lambda}, \frac{1}{\lambda}\right]$, gives

$$
v(t, x, y)=(x+y)^{p} \phi(t, \xi), \quad(t, \xi) \in[0, T] \times Q_{v} .
$$

Theorem $2 \phi$ is a viscosity solution of the HJB equation $\mathcal{L}(\phi)=0$ on $[0, T] \times \overline{Q_{\phi}}$;

$$
\mathcal{L}(\phi)=\min \left\{-\phi_{t}-\mathcal{P}(\phi), \lambda p \phi(t, \xi)+(1-\lambda \xi) \phi_{\xi}(t, \xi), \lambda p \phi(t, \xi)-(1+\lambda \xi) \phi_{\xi}(t, \xi)\right\}
$$


such that

$$
\begin{aligned}
\mathcal{P}(\phi) & =\left\{-\rho+p\left(\mu(1-\xi)+\mu_{2} \xi\right)+\frac{p(p-1)}{2}\left(\sigma_{2}^{2} \xi^{2}+\sigma_{1}^{2}(1-\xi)^{2}+2 \beta \sigma_{1} \sigma_{2} \xi(1-\xi)\right)\right\} \phi(t, \xi) \\
& +\xi(1-\xi)\left[\mu_{2}-\mu+(p-1)\left(\beta \sigma_{1} \sigma_{2}(1-2 \xi)+\xi\left(\sigma_{1}^{2}+\sigma_{2}^{2}\right)\right)\right] \phi_{\xi}(t, \xi) \\
& -\frac{\xi^{2}(1-\xi)^{2}}{2}\left(\sigma_{2}^{2}+\sigma_{1}^{2}+2 \beta \sigma_{1} \sigma_{2}\right) \phi_{\xi \xi}(t, \xi) \\
& -\int_{\mathbb{R}}\left\{\phi(t, \xi+h(\xi, \zeta))-\phi(t, \xi)(1+p h(\xi, \zeta))-\phi_{\xi}(t, \xi) h(\xi, \zeta)\right\} v(d \zeta), \\
& =\left\{p A-\Lambda_{0}+\frac{p(p-1)}{2}\left(\left({\sigma_{1}}^{2}+\sigma_{2}^{2}\right)(\xi-\pi)^{2}+2 \beta \sigma_{1} \sigma_{2} \xi(1-\xi)\right)\right\} \phi(t, \xi) \\
& +(p-1) \xi(1-\xi)\left[(\xi-\pi)\left(\sigma_{1}^{2}+\sigma_{2}^{2}\right)+\beta \sigma_{1} \sigma_{2}(1-2 \xi)+\sigma_{1}^{2}+\int_{\mathbb{R}} g^{2}(t, \zeta) v(d \zeta)\right] \phi_{\xi}(t, \xi) \\
& -\frac{\xi^{2}(1-\xi)^{2}}{2}\left(\sigma_{2}{ }^{2}+\sigma_{1}{ }^{2}+2 \beta \sigma_{1} \sigma_{2}\right) \phi_{\xi \xi}(t, \xi) \\
- & \int_{\mathbb{R}}\left\{\phi(t, \xi+h(\xi, \zeta))-\phi(t, \xi)(1+p h(\xi, \zeta))-\phi_{\xi}(t, \xi) h(\xi, \zeta)\right\} v(d \zeta),
\end{aligned}
$$

where

$$
\begin{aligned}
h(\xi, \zeta) & =(x+y)^{-1} g(y, \zeta) \\
& =(x+y)^{-1} y k(t, \zeta) \\
& =\xi k(t, \zeta),
\end{aligned}
$$

and

subject to the terminal condition

$$
\Lambda_{0}=\int_{\mathbb{R}}\left\{\left(1+\pi^{\star} g(t, \zeta)\right)^{p}-1+p \pi^{\star} g(t, \zeta)\left((p-1) \pi^{\star} g(t, \zeta)-1\right)\right\} v(d \zeta),
$$

$$
\phi(T, \xi)=U(1-\lambda|\xi|), \quad \xi \in \overline{Q_{\phi}} .
$$

For convenience we shall from now on impose the following additional integrability condition on $v(\cdot)$ :

$$
\int_{\mathbb{R}} g^{2}(t, \zeta) v(d \zeta)<\infty
$$

\section{Remark}

1. This is equivalent to Bichuch (2011) when there are no jump term and no foreign exchange fluctuations ( that is, $\sigma_{1}=0$ and $\mu_{1}=0$ ).

2. In the presence of the jumps, we then study the scenario when the impact of the foreign exchange is given by

$$
\begin{aligned}
\Lambda_{0} \phi(t, \xi) & =\left((p-1) \xi(1-\xi) \int_{\mathbb{R}} g^{2}(t, \zeta) v(d \zeta)\right) \phi_{\xi}(t, \xi) \\
& -\int_{\mathbb{R}}\left\{\phi(t, \xi+h(\xi, \zeta))-\phi(t, \xi)(1+p h(\xi, \zeta))-\phi_{\xi}(t, \xi) h(\xi, \zeta)\right\} v(d \zeta) .
\end{aligned}
$$

We will be concerned with the nature of the solution to equation (26) as the impact of foreign exchange. We assume $\phi(t, \xi)=f(\xi) l(t)$ and $g(t, \zeta)=\zeta$. Since $l(t) \neq 0$, then (26) simplifies to

$$
\begin{aligned}
\int_{\mathbb{R}} f(\xi(1+\zeta)) v(d \zeta) & =\left((p-1) \xi(1-\xi) \int_{\mathbb{R}} \zeta^{2} v(d \zeta)+\xi \int_{\mathbb{R}} v(d \zeta)\right) f_{\xi}(\xi) \\
& +\left(\int_{\mathbb{R}}(1-p \xi \zeta) v(d \zeta)-\Lambda_{0}\right) f(\xi) .
\end{aligned}
$$

For the ease of the computations, we set the following:

$$
\int_{\mathbb{R}} f(\xi(1+\zeta)) v(d \zeta)=0
$$


so we have

$$
\begin{aligned}
f(\xi) & =A_{0} \exp \left\{\int_{\overline{Q_{\phi}}} \frac{\int_{\mathbb{R}}(1-p \xi \zeta) v(d \zeta)-\Lambda_{0}}{\left(\int_{\mathbb{R}}\left((1-p)(1-\xi) \zeta^{2}+1\right) v(d \zeta)\right) \xi} d \xi\right\} \\
& =A_{0} \exp \left\{R \log (\xi)+P \log \left(\int_{\mathbb{R}}\left((1-p)(1-\xi) \zeta^{2}+1\right) v(d \zeta)\right)\right\}
\end{aligned}
$$

where

$$
\begin{gathered}
R=\frac{\int_{\mathbb{R}} v(d \zeta)-\Lambda_{0}}{\int_{\mathbb{R}}\left((1-p) \zeta^{2}+1\right) v(d \zeta)} \\
P=\frac{\Lambda_{0}-\int_{\mathbb{R}} v(d \zeta)}{\int_{\mathbb{R}}\left((1-p) \zeta^{2}+1\right) v(d \zeta)}+\frac{p \int_{\mathbb{R}} v(d \zeta)}{(1-p) \int_{\mathbb{R}} \zeta^{2} v(d \zeta)}
\end{gathered}
$$

and $A_{0}$ is a constant. Satisfying the following condition

$$
\int_{\mathbb{R}}\left((1-p)(1-\xi) \zeta^{2}+1\right) v(d \zeta)>0 .
$$

We need to specify the Lévy measure $v(d \zeta)$ driving the common jumps; then we can successfully compute the integrals in (27). This is considered under different cases below:

\subsection{Uniform Jumps}

Proposition 1 Suppose the value function is $\phi(t, \zeta)=f(\zeta) l(t), l(t) \neq 0$ and $g(t, \zeta)=\zeta$, then under uniform jumps, (28) is valid if

$$
0<\xi<\left.\frac{(1-p) \zeta^{2}+3}{(1-p) \zeta^{2}}\right|_{\mathbb{R}} .
$$

Proof. Set $v(d \zeta)=\tau d \zeta$, where $\tau$ is arbitrary constant describing the intensity of the jump. Then we obtain

$$
\begin{aligned}
\int_{\mathbb{R}} f(\xi(1+\zeta)) d \zeta & =\left.\left(\frac{p-1}{3}(1-\xi) \zeta^{2}+1\right) \xi \zeta\right|_{\mathbb{R}} f_{\xi}(\xi) \\
& +\left(\left.(1-p \xi \zeta) \zeta\right|_{\mathbb{R}}-\Lambda_{0}^{\star}\right) f(\xi)
\end{aligned}
$$

where

$$
\Lambda_{0}^{\star}=\left.\left(\frac{(1+\pi \zeta)^{1+p}}{(1+p) \pi}-\frac{p(1-p)}{3} \pi^{2} \zeta^{3}+\frac{p}{2} \pi \zeta^{2}+\zeta\right)\right|_{\mathbb{R}} .
$$

Assuming that the integral on the right of (30) varnishes, we then obtain the following solution

$$
f(\xi)=B_{0} \exp \left\{\int_{\overline{Q_{\phi}}} \frac{\left.(1-p \xi \zeta) \zeta\right|_{\mathbb{R}}-\Lambda_{0}^{\star}}{\left.\left(\frac{1-p}{3}(1-\xi) \zeta^{2}+1\right) \xi \zeta\right|_{\mathbb{R}}} d \xi\right\}
$$

where $B_{0}$ is a constant of integration and

$$
\begin{aligned}
& \left.\int \frac{\left.(1-p \xi \zeta) \zeta\right|_{\mathbb{R}}-\Lambda_{0}^{\star}}{\overline{Q_{\phi}}} d \xi=\left.P^{\star}(\zeta) \log \left(\frac{1-p}{3}(1-\xi) \zeta^{2}+1\right) \xi \zeta\right|_{\mathbb{R}}(1-\xi) \zeta^{2}+1\right)\left.\right|_{\mathbb{R}} \\
& +\left.R^{\star}(\zeta) \log (\xi)\right|_{\mathbb{R}},
\end{aligned}
$$

where

$$
R^{\star}(\zeta)=\frac{3\left(1-\Lambda_{0}^{\star}\right)}{(1-p) \zeta^{2}+3}
$$


and

such that the inequality

$$
P^{\star}(\zeta)=\frac{3\left(\Lambda_{0}^{\star}-\zeta\right)}{\left((1-p) \zeta^{2}+3\right) \zeta}+\frac{3 p}{(1-p) \zeta} .
$$

is met.

\subsection{Power Law Jumps}

We assume that the arrival of jumps of size $\zeta$ follows a power law, dampened by an exponential function:

$$
v(d \zeta)=\left\{\begin{array}{l}
\tau^{+} e^{-\beta_{+} \zeta} \zeta^{-\alpha-1} d \zeta, \text { if } \zeta \in(0, \infty) \\
-\tau^{-} e^{-\beta-|\zeta|}|\zeta|^{-\alpha-1} d \zeta, \text { if } \zeta \in(-\infty, 0),
\end{array}\right.
$$

with the parameters $\alpha \in[-1,2)$ and $\beta_{ \pm}, \tau^{ \pm} \in \mathbb{R}^{+}$. Setting $\beta_{ \pm}=0$, that is, without exponential dampening, the Lévy density uniquely determines an $\alpha$-stable Lévy motion that generates the $\alpha$-stable distribution (see Mandelbrot (1963) and Fama (1965)). For strictly dampening $\beta_{ \pm}>0$, the exponential functions $e^{-\beta_{+} \zeta}$ and $e^{-\beta_{-}|\zeta|}$ dampen the Lévy density so that the arrival of jumps decays faster as the absolute jump size $|\zeta|$ increases (see Wu (2006)). We also note the following:

1. When $\alpha<0: \int_{\mathbb{R}} v(d \zeta)<0$. Then we have large and rare events.

2. Infinite activity when $\alpha \geq 0$ : Both small and large jumps. Jumps frequency increases with declining jump size and approaches infinity as $\zeta \rightarrow 0$.

3. Infinite Variation when $\alpha \geq 1$ : many small jumps.

We shall consider a special case where the exponential dampening parameter, $\beta=1$, and propose as follows;

Proposition 2 Suppose the value function is $\phi(t, \xi)=f(\xi) l(t), l(t) \neq 0$ and $g(t, \zeta)=\zeta, t \in[0, T]$, then (28) is valid under dampen power law jumps with $\beta^{ \pm}=1$ if

$$
0<\xi<\frac{(1-p)(1-\alpha) \alpha-1}{(1-p)(1-\alpha) \alpha} \text {. }
$$

Proof. Setting $\beta^{ \pm}=1, \tau^{ \pm}=\tau>0$ and $\alpha \in[-1,2)$ in (35). Then for all $t \in[0, T]$, the value function is given by

$$
\phi(t, \xi)=A_{0} l(t) \exp \{R \log (\xi)+P \log (2 \tau \Gamma(-\alpha)(1-\alpha(1-\alpha)(1-p)(1-\xi)))\}
$$

where $\Gamma(\cdot)$ is a gamma function,

$$
\begin{gathered}
R=\frac{1-\pi((1-p) \pi \alpha-p)(1-\alpha)}{1-(1-p)(1-\alpha) \alpha}, \\
P=\frac{p}{(p-1)(1-\alpha) \alpha}-R,
\end{gathered}
$$

and $A_{0}$ is an arbitrary constant. The value function $\phi(t, \xi)$ is defined only when

$$
0<\xi<\frac{(1-p)(1-\alpha) \alpha-1}{(1-p)(1-\alpha) \alpha} \text {. }
$$

Which completes the short proof.

\subsection{Fixed Jump Size}

Proposition 3 Suppose the value function is $\phi(t, \xi)=f(\xi) l(t), l(t) \neq 0$ and $g(t, \zeta)=\zeta$, then (28) is valid under fixed jump size if

$$
0<\xi<\frac{11-2 p}{2(1-p)}
$$

Proof. Set $v(d \zeta)=\delta(\zeta=\bar{\zeta}) d \zeta$, for some $\bar{\zeta} \in[-1,1]$ as the Dirac measure. This measure is associated to a point $\zeta \in \mathbb{R}$ defined as follows:

$$
v(d \zeta)= \begin{cases}d \zeta, & \zeta \in[-1,1] \\ 0, & \zeta \notin[-1,1]\end{cases}
$$


It then follows that (28) becomes

$$
f(\xi)=\left\{\begin{array}{l}
A_{0} \exp \left\{\frac{3\left(2-\Lambda_{2}\right)}{2(1-p)} \log (\xi)+\frac{(1-p) \Lambda_{2}-2\left(p^{2}-3 p+1\right)}{(2-p)(1-p)} \log \left(\frac{2}{3}((1-p)(1-\xi)+3)\right)\right\}, \quad \zeta \in[-1,1] \\
0, \quad \zeta \notin[-1,1] .
\end{array}\right.
$$

where

$$
\Lambda_{2}= \begin{cases}\frac{1}{(1+p) \pi}\left((1+\pi)^{1+p}-(1-\pi)^{1+p}\right)-\frac{2}{3}\left((1-p) \pi^{2}+3\right), & \zeta \in[-1,1] \\ 0, & \zeta \notin[-1,1]\end{cases}
$$

$A_{0}$ is a constant and

$$
0<\xi<\frac{11-2 p}{2(1-p)}
$$

Since the jump sizes are fixed, it becomes too restrictive and less suitable to use in modelling stock price (for instance). But it can be used as a building block to construct richer models.

\section{Results}

Even though the stock price may experience some jumps, if they are small in absolute value then the total payoff increases exponentially. It is also observed that in a jump setting, if the jump measure is zero then we have Merton model setting and optimal portfolio matches. The effect of jumps, transaction costs and foreign exchange is significant as it makes the model realistic.

\section{References}

Applebaum, D. (2004). Lévy Processes and Stochastic Calculus. Cambridge: Cambridge University Press. https://doi.org/10.1017/CBO9780511755323

Bichuch, M. (2011). Asymptotic Analysis for Optimal Investment in Finite Time with Transaction Costs. J. Financial Math., 3(1), 433-458. https://doi.org/10.1137/100808046

Davis, M. H. A., \& Norman, A. (1990). Portfolio Selection with Transaction Costs. Math. Operations Research, 15, 676-713. https://doi.org/10.1287/moor.15.4.676

Davis, M. H. A., Panas, V. G., \& Zariphopoulou, T. (1993). Europian Option Pricing with Transaction Costs. SIAM J. Control and Optimization, 3l(2), 470-493. https://doi.org/10.1137/0331022

Fama, E. F. (1965). Risk minimizing portfolios and HJBI equations for stochastic differential games. Journal of Business 38, 34-105. https://doi.org/10.1086/294743

Fleming, W. H., \& Soner, H. T. (1993). Controlled Markov Processes and Viscosity Solutions. Springer-Verlag. New York.

Janeček, K., \& Shreve, S. E. (2004). Asymptotic Analysis for Optimal Investment and Consumption with Transaction Costs. Financial Stoch., 8(2), 181-206. https://doi.org/10.1007/s00780-003-0113-4

Lévy, P. (1937). Théories de LAddition Aléatories. Paris: Gauthier-Villars.

Liu, H. (2004). Optimal Consumption and Investment with Transaction Costs and Multiple Risky Assets. J. Finance, 59(1), 289-338. https://doi.org/10.1111/j.1540-6261.2004.00634.x

Mandelbrot, B. B. (1963). The variation of certain speculative prices. Journal of Business 36, 394-419. https://doi.org/10.1086/294632

Merton, R. (1971). Optimum Consumption and Portfolio Rules in a Continuous-time Case. J. Economic Theory, 3, 373-413. https://doi.org/10.1016/0022-0531(71)90038-X

Øksendal, B., \& Sulem, A. (2004). Optimal Consumption and Portfolio with both fixed and Proportional Transaction Costs. SIAM J. Control Optimization, 40(6), 1765-1790. https://doi.org/10.1137/S0363012900376013

Øksendal, B., \& Sulem, A. (2005). Applied Stochastic Control of Jump Diffusions. Springer-Verlag Berlin Heidelberg.

Øksendal, B., \& Sulem, A. (2014). Stochastic Control of Ito-Lévy Processes with Applications to Finance. Communications on Stochastic Analysis 8(1), 1-15.

Sato K. (1999). Lévy Processes and Infinitely Divisible Distributions. Cambridge: Cambridge University Press.

Shreve S. E., \& Soner H. M. (1994). Optimal Investment and Consumption with Transaction Costs. Ann. Applied Probab., 4, 609-692. https://doi.org/10.1214/aoap/1177004966 
Tankov, P. (2005). Financial Modelling with Lévy Processes: Lecture Notes. Ecole Polytechnique.

Wu, L. (2006). Dampened power law: Reconciling the tail behavior of financial security returns. Journal of Business, 79(3).

\section{Copyrights}

Copyright for this article is retained by the author(s), with first publication rights granted to the journal.

This is an open-access article distributed under the terms and conditions of the Creative Commons Attribution license (http://creativecommons.org/licenses/by/4.0/). 